\title{
Ligninolytic Peroxidase-Like Activity of a Synthetic Metalloporphine Immobilized onto Mercapto-Grafted Crosslinked PVA Inspired by the Active Site of Cytochrome P450
}

\author{
Paolo ZUCCA ${ }^{1, *}$, Antonio RESCIGNO ${ }^{1,2}$, Enrico SANJUST ${ }^{1,2}$ \\ ${ }^{\prime}$ Dipartimento di Scienze e Tecnologie Biomediche, Università di Cagliari, Monserrato 09042, Italy \\ ${ }^{2}$ Consorzio per lo Sviluppo dei Sistemi a Grande Interfase, Sesto Fiorentino 50019, Italy
}

\begin{abstract}
A synthetic metalloporphine was immobilized onto a PVA-based and mercapto-grafted solid support, emulating the active site of cytochrome P450. Its ligninolytic peroxidase-like catalytic activity was studied. The coordinated mercapto ligand significantly affected the catalytic features of the catalyst because the oxidation of lignin-model compounds was very slow by comparison with imidazole- and pyridine-coordinated immobilized metalloporphines. Conversely, the catalyst efficiently bleached several industrial dyes and thus demonstrated promising activity for this application. Based on this altered substrate specificity the oxygen-donor catalytic route seems to be more favorable than a single electron oxidation pathway.
\end{abstract}

Key words: porphine; porphyrin; lignin peroxidase; cytochrome P450; biomimetic

CLC number: O643 Document code: A

Received 2 August 2011. Accepted 13 September 2011.

*Corresponding Author. Tel: +39-070675-4514; Fax+39-070675-4527; E-mail address: pzucca@unica.it

This work was supported by the Regione Autonoma Sardegna (PO Sardegna FSE 2007-2013, L.R.7/2007, Bando Giovani Ricercatori, Project CRP1_27).

English edition available online at Elsevier ScienceDirect (http://www.sciencedirect.com/science/journal/18722067).

Recently, catalysts that promote oxidative delignification under mild conditions have attracted interest. Synthetic metalloporphines have proven to be promising catalysts for this reaction because they are able to oxidize lignin-related molecules [1] and other pollutant (and industrially relevant) substrates [2,3]. In particular, the structural and functional emulation of lignin peroxidase has been described [4] using imidazole as a coordinating group for metalloporphines. This catalyst showed promising catalytic features and worked well under very mild conditions. Based on these promising results, other coordinating groups (such as pyridine [5]) have been studied for the synthesis of immobilized metalloporphine-based catalysts. Herein, we report on the immobilization of a synthetic metalloporphine onto a mercapto-grafted solid support to emulate the structure of the active site of cytochrome P450. Cytochrome P450 is a large superfamily of hemoproteins that catalyze monooxygenase reactions [6,7] with $\mathrm{O}_{2}$ as an oxidizing agent and NADPH as a co-reducing agent. In this enzyme the mercapto group of a cysteine residue is present in the active site as the proximal ligand of ferriheme [8]. The ability of metalloporphines to emulate P450 catalysis during monooxygenase reactions has been thoroughly studied [9-11]. In this paper, we describe the influence of the mercapto ligand during the ligninolytic peroxidase-like catalysis of immobilized metalloporphine in the presence of $\mathrm{H}_{2} \mathrm{O}_{2}$. To our knowledge, no studies exist regarding similar characterizations of metalloporphines immobilized on mercapto-functionalized supports.

All reagents were of the best commercial grade available and were used without further purification. ARS was purchased from Fluka (cat. No. 05600), FeTFPP from Aldrich (cat. No. 252913), lignin peroxidase from Sigma-Aldrich (cat. No. 42603), and PVA from Aldrich (cat. No. 363138). Laccase from $P$. sajor-caju was purified by two chromatographic steps (details in [12]).

Spectrophotometric measurements were carried out with an UltroSpec 2100pro (Amersham Bioscience). The concentrations of the substrates were also measured by UV-HPLC and GC-MS using previously described methods $[4,13]$. Amino-grafted PVA (AP-PVA) was synthesized as previously described [5,14].

Each gram of the AP-PVA powder was suspended in 10 $\mathrm{ml}$ of a $0.1 \mathrm{~mol} / \mathrm{L}$ aqueous solution of $\mathrm{NaHCO}_{3}$ containing $0.5 \mathrm{~g} N$-acetyl-DL-homocysteine thiolactone. The slurry was then stirred gently for $24 \mathrm{~h}$ at $25{ }^{\circ} \mathrm{C}$. The obtained M-PVA was then consecutively washed with $\mathrm{H}_{2} \mathrm{O}, 0.1 \mathrm{~mol} / \mathrm{L}$ aqueous $\mathrm{KH}_{2} \mathrm{PO}_{4}, \mathrm{H}_{2} \mathrm{O}$ again, $0.1 \mathrm{~mol} / \mathrm{L}$ aqueous solution of 
sodium dithionite, $\mathrm{H}_{2} \mathrm{O}$ again, and 2-propanol.

The mercapto-grafted PVA was then carefully dried overnight in a vacuum oven at $50{ }^{\circ} \mathrm{C}$. To determine the amount of aminopropyl moieties and the extent of their further derivatization with mercapto functions, a chromogenic reaction with ninhydrin and hydrindantin was applied [15].

One-gram aliquots of M-PVA were treated with $20 \mathrm{mg}$ of FeTFPP solubilized in $10 \mathrm{ml}$ of DMSO. The slurry was kept in the dark for $24 \mathrm{~h}$ while stirring and washed repeatedly with DMSO and 2-propanol. Finally, the adduct was dried at $50{ }^{\circ} \mathrm{C}$. The proposed structure of the obtained catalyst is shown in Fig. 1. Bound metalloporphine was quantified by differences in the spectrophotometric measurements at 411 $\mathrm{nm}\left(\varepsilon_{411}=115000 \mathrm{~mol} /(\mathrm{L} \cdot \mathrm{cm})[5]\right)$.

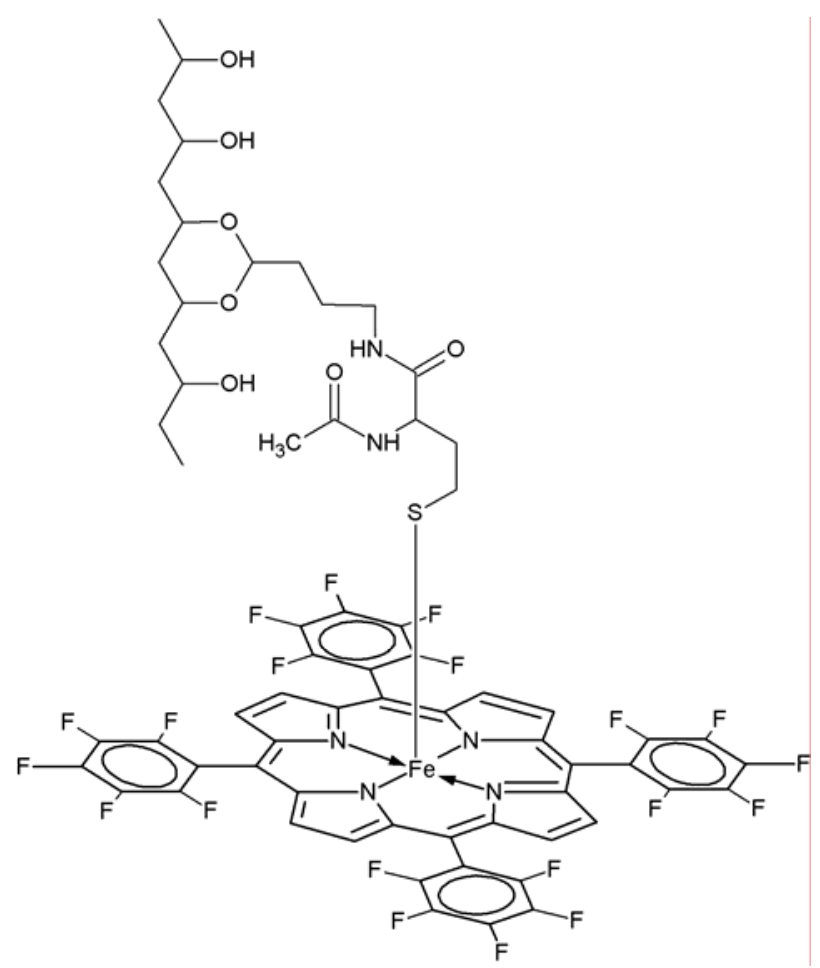

Fig. 1. Proposed structure of the catalytic adduct M-PVA/FeTFPP.

Routine measurements of the catalytic activity of immobilized metalloporphine were performed by spectrophotometric assays. A suspension of $5 \mathrm{mg}$ of catalyst in $1 \mathrm{ml}$ of $25 \mathrm{mmol} / \mathrm{L}$ buffer solution containing $12 \mathrm{mmol} / \mathrm{L}$ VA and $8.8 \mathrm{mmol} / \mathrm{L}$ hydrogen peroxide was stirred at $25{ }^{\circ} \mathrm{C}$ in the dark. After $30 \mathrm{~min}$, the increase in absorbance at $310 \mathrm{~nm}$ was measured to detect the formed veratraldehyde $\left(\varepsilon_{310}=\right.$ $9300 \mathrm{~mol} /(\mathrm{L} \cdot \mathrm{cm})$ [16]). In the case of ARS the reducing substrate concentration was $1.2 \mathrm{mmol} / \mathrm{L}$ and the absorbance decrease at $520 \mathrm{~nm}$ was recorded $\left(\varepsilon_{520}=7200 \mathrm{~mol} /(\mathrm{L} \cdot \mathrm{cm})\right.$ [17]).

In some experiments the well-known hydroxyl radical scavenger $0.1 \mathrm{~mol} / \mathrm{L}$ mannitol was also added. When used, redox mediators (OH-TEMPO, NPH, NHS, and NHT) were present in the reaction medium at a final concentration of 1 $\mathrm{mmol} / \mathrm{L}$.

To test the catalytic performance at various $\mathrm{pH}$ levels, different McIlvaine buffers were used at $\mathrm{pH}=3,4,5,6,7$, and 8.

Other compounds were also tested as substrates, including 4-methoxybenzyl alcohol, 4-hydroxy-3- methoxybenzyl alcohol (vanillyl alcohol), 3-hydroxy-4-methoxybenzyl alcohol (isovanillyl alcohol), 3,4,5-trimethoxybenzyl alcohol, 3,4-dimethoxybenzaldehyde (veratraldehyde), 3,4- dimethoxybenzoic acid (veratric acid), 1,2-dimethoxybenzene (veratrole), 1,2,3-trimethoxybenzene, and 4-hydroxy-3methoxycinnamic acid (ferulic acid).

For the MnP-like assays all the experiments were repeated in aqueous media with $1 \mathrm{mmol} / \mathrm{L} \mathrm{MnSO}_{4}$ and 50 $\mathrm{mmol} / \mathrm{L}$ malonic acid. For the multicyclic runs, the catalyst was regenerated between consecutive cycles by repeated washing with water and 2-propanol and by drying at $50{ }^{\circ} \mathrm{C}$.

Inexpensive and hydrophilic PVA was efficiently grafted with mercapto functional groups (280 $\mu \mathrm{mol} / \mathrm{g}$ of support) using the well-known reaction between amines and $\mathrm{N}$-acetyl-DL-homocysteine thiolactone. This reaction also allowed for the insertion of a long and flexible molecular spacer arm between the support and the catalyst.

After being grafted the support was immediately treated with metalloporphine to avoid the possible oxidation of the -SH group. FeTFPP was chosen for this study because of its notable catalytic efficiency and chemical stability compared with its physiological counterpart ferriprotoporphyrin IX [18]. The metalloporphine bound to the support gave a typical saturation curve (not shown) but the highest specific catalytic activity was observed when $20 \mathrm{mg}$ of FeTFPP per $\mathrm{g}$ of M-PVA was added (leading to an effective binding of $8.8 \mathrm{mg}$ or $8.3 \mu \mathrm{mol})$. Accordingly, this FeTFPP concentration was chosen for further study.

Significantly, in the presence of $\mathrm{O}_{2}$ and $\mathrm{NADPH}$, M-PVA/FeTFPP was unable to act as a monooxygenase for a variety of possible oxygen acceptors.

However, when $\mathrm{H}_{2} \mathrm{O}_{2}$ was present in the reaction mixture, M-PVA/FeTFPP catalyzed the desired peroxidase-like reactions of both VA and ARS.

For VA oxidation the observed catalytic activity was quite poor ( $1 \mathrm{~h}$ provided $17 \%$ conversion of VA) by comparison with similar metalloporphines immobilized with different ligands $[4,5]$. The product distribution remained similar: veratraldehyde was only obtained with $21 \%$ selectivity along with byproducts that were unidentifiable by UV-HPLC and GC-MS (only very small, non-quantifiable amounts of 2-hydroxymethyl-5-methoxy-1,4-benzoquinone and veratric acid were observed).

For ARS oxidation, however, a significantly higher conversion was observed (over $80 \%$ in $1 \mathrm{~h}$ ). This behavior 
could be rationalized by considering that VA can only be oxidized by a single electron transfer pathway whereas ARS (or other ARS partial oxidation-derived species) can act as a substrate for monooxygenase-like reactions [3].

The ability of metalloporphines to act as both monoelectronic oxidants and as oxygen donors has previously been described [3]. Our results suggest that the mercapto coordinating group is able to make the latter route more favorable in accordance with the attempted structural emulation. In fact, ligninolytic peroxidases that present imidazoles (of a specific histidine residue) as proximal coordinating groups are only able to achieve monoelectronic oxidation. Thus, supported metalloporphines that resemble their active sites are capable of catalyzing the nearly complete conversion of substrates such as VA. Mercapto coordinating groups, however, can change the activity of the metalloporphine oxygen-donor ability as in cytochrome P450.

The effects of $\mathrm{Mn}^{2+}$ on the catalytic process were also studied. In its absence, the M-PVA/FeTFPP adduct showed a $\mathrm{pH}$ optimum of 3 for both VA and ARS (Fig. 2). In the presence of $\mathrm{Mn}^{2+}$, however, VA oxidation values increased whereas ARS oxidation seemed to be inhibited by $\mathrm{Mn}^{2+}$.

The catalyst was also characterized kinetically, showing a typical Michaelis-Menten saturation for both substrates. A relatively high $K_{\mathrm{m}}$ value $(7.6 \pm 2.5 \mathrm{mmol} / \mathrm{L})$ confirmed the low specificity of M-PVA/FeTFPP for VA while the $K_{\mathrm{m}}$ for ARS $(1.12 \pm 0.02 \mathrm{mmol} / \mathrm{L})$ was similar to those observed in

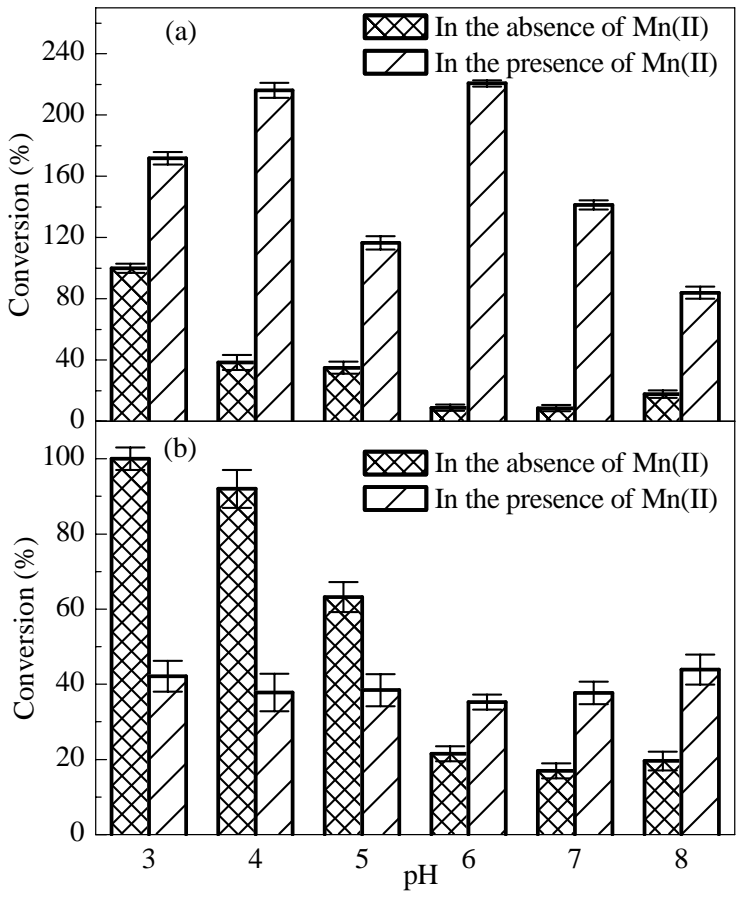

Fig. 2. Relative activity of the M-PVA/FeTFPP adduct toward VA (a) and ARS (b) at various $\mathrm{pH}$ values. the presence of ligands other than the mercapto group [3,5].

The poor LiP-like performances of M-PVA/FeTFPP were confirmed during oxidation screenings of various lignin-model compounds (Table 1). The conversions were very low for both phenolic and non-phenolic substrates, especially upon comparison with similar experiments containing pyridine as the FeTFPP ligand [5].

Table 1 Substrate specificity of the M-PVA/FeTFPP adduct with various lignin model compounds

\begin{tabular}{lc}
\hline Substrate & Conversion (\%) \\
\hline Veratryl alcohol & 18.0 \\
Veratraldehyde & 9.9 \\
Veratric acid & 2.7 \\
Isovanillyl alcohol & 29.6 \\
Vanillyl alcohol & 46.2 \\
1,2,3-Trimetoxybenzene & 0 \\
Ferulic acid & 86.2 \\
Veratrole & 18.2 \\
4-Methoxybenzyl alcohol & 10.6 \\
3,4,5-Trimethoxybenzyl alcohol & 1.8 \\
\hline
\end{tabular}

Catalytic activity was detected in the presence of $5 \mathrm{mg}$ of catalyst, 8.8 mmol/L hydrogen peroxide, $1 \mathrm{mmol} / \mathrm{L}$ substrate, DME $10 \%, 1 \mathrm{mmol} / \mathrm{L}$ $\mathrm{MnSO}_{4}, 50 \mathrm{mmol} / \mathrm{L}$ malonic acid, and $25 \mathrm{mmol} / \mathrm{L}$ buffer $(\mathrm{pH}=4$, final volume $2 \mathrm{ml})$.

A homolytic cleavage of the $\mathrm{O}-\mathrm{O}$ bond was excluded as a possible pathway [19] because mannitol (a well-known $\mathrm{OH} \bullet$ scavenger) did not affect the catalytic properties of the adduct.

Additional redox mediators were also tested: OH-TEMPO, NPH, NHS, and NHT. ARS bleaching was not greatly affected by their presence and the rate of VA oxidation was tripled in the presence of OH-TEMPO and NHS.

According to all the data reported above, M-PVA/FeTFPP is not a good LiP emulator. This finding was further corroborated by a multicycle analysis. For VA oxidation, only $7 \%$ catalytic activity was obtained in the second cycle and for subsequent cycles no activity was observed. More promising results, however, were obtained for ARS since about $20 \%$ of the catalytic activity was obtained after 6 cycles.

In addition to ARS, the ability of M-PVA/FeTFPP to bleach a complete series of dyes belonging to different chemical classes was also tested (Table 2). The conversion rates over $1 \mathrm{~h}$ were quite high for almost all the dyes tested and ranged from $50 \%$ to $90 \%$ (only XO conversion was low at $23 \%$ ). However, in all cases the adduct was catalytically active confirming the wide substrate specificity of the immobilized metalloporphine, particularly when pathways apart from single electron oxidation are feasible.

The substrate specificity with industrial dyes was even broader than that of ligninolytic enzymes. For example, 
Table 2 M-PVA/FeTFPP adduct bleaching activity with several dyes

\begin{tabular}{lc}
\hline Dye & Conversion (\%) \\
\hline ARS & 83 \\
$0.3 \mathrm{mmol} / \mathrm{L} \mathrm{PNS}$ & 59 \\
$2 \mathrm{mmol} / \mathrm{L} \mathrm{XO}$ & 23 \\
$0.15 \mathrm{mmol} / \mathrm{L} \mathrm{MB}$ & 50 \\
$1.5 \mathrm{mmol} / \mathrm{L} \mathrm{MG}$ & 47 \\
$1.2 \mathrm{mmol} / \mathrm{L} \mathrm{MO}$ & 97 \\
\hline
\end{tabular}

Catalytic activity was detected in the presence of $5 \mathrm{mg}$ of catalyst, 8.8 $\mathrm{mmol} / \mathrm{L}$ hydrogen peroxide and $25 \mathrm{mmol} / \mathrm{L}$ buffer $(\mathrm{pH}=4$, final volume $2 \mathrm{ml}$ ). The dyes were present at a concentration of $0.3 \mathrm{mmol} / \mathrm{L}$ PNS, $2 \mathrm{mmol} / \mathrm{L} \mathrm{XO}, 0.15 \mathrm{mmol} / \mathrm{L}$ methylene blue, $1.5 \mathrm{mmol} / \mathrm{L}$ methyl green, and $1.2 \mathrm{mmol} / \mathrm{L}$ methyl orange.

laccase and lignin peroxidases under similar operational conditions gave lower conversion rates [20] and were completely unable to oxidize some of the dyes such as PNS.

In conclusion, the use of a mercapto group to immobilize metalloporphines did not allow for an efficient ligninolytic peroxidase-like emulation with lignin-model compounds (contrary to what has been observed with imidazole and pyridine as ligands of similar metalloporphines). However, the M-PVA/FeTFPP adduct showed interesting features in the treatment of several industrial dyes that were efficiently bleached under very mild operational conditions. These results are very promising, particularly from an industrial application point of view. Furthermore, the collected data suggests that the mercapto group can change the catalytic mechanism of FeTFPP since its oxygen-donor behavior becomes more favorable than its single electron transfer behavior.

\section{References}

1 Crestini C, Pastorini A, Tagliatesta P. J Mol Catal A, 2004,
208: 195

2 Zucca P, Vinci C, Rescigno A, Dumitriu E, Sanjust E. J Mol Catal A, 2010, 321: 27

3 Zucca P, Vinci C, Sollai F, Rescigno A, Sanjust E. J Mol Catal A, 2008, 288: 97

4 Zucca P, Mocci G, Rescigno A, Sanjust E. $J$ Mol Catal A, 2007, 278: 220

5 Zucca P, Sollai F, Garau A, Rescigno A, Sanjust E. $J$ Mol Catal A, 2009, 306: 89

6 Ge X, Wang J, Liu J, Jiang J, Lin H, Wu J, Ouyang M, Tang X, Zheng M, Liao M, Deng Y. Catal Commun, 2010, 12: 71

7 Sigel A, Sigel H, Sigel R K O. The Ubiquitous Roles of Cytochrome P450 Proteins. Chichester: Wiley, 2007

8 Tani F, Matsu-ura M, Nakayama S, Naruta Y. Coord Chem Rev, 2002, 226: 219

9 Costa A A, Ghesti G F, de Macedo J L, Braga V S, Santos M M, Dias J A, Dias S C L. J Mol Catal A, 2008, 282: 149

10 Ghiaci M, Molaie F, Sedaghat M E, Dorokstar N. Catal Commun, 2010, 11: 694

11 Mansuy D C R. Chimie, 2007, 10: 392

12 Rescigno A, Zucca P, Flurkey A, Inlow J, Flurkey W H. Enzyme Microb Technol, 2007, 41: 620

13 Zucca P, Littarru M, Rescigno A, Sanjust E. Biosci Biotechnol Biochem, 2009, 73: 1224

14 Sanjust E, Curreli N, Maullu C, Rinaldi A, Rescigno A. Materials Engineering, 1994, 5: 69

15 Filippo P A D, Fadda M B, Rescigno A, Rinaldi A, Di Teulada E S. Eur Polym J, 1990, 26: 545

16 Sarkanen S, Razal R A, Piccariello T, Yamamoto E, Lewis N G. J Biol Chem, 1991, 266: 3636

17 Lachheb H, Puzenat E, Houas A, Ksibi M, Elaloui E, Guillard C, Hermann J M. Appl Catal B, 2002, 39: 75

18 Traylor T G, Hill K W, Fann W P, Tsuchiya S, Dunlap B E. $J$ Am Chem Soc, 1992, 114: 1308

19 Meunier B. Chem Rev, 1992, 92: 1411

20 Zucca P, Rescigno A, Olianas A, Maccioni S, Sollai F A, Sanjust E. J Mol Catal B, 2011, 68: 216 